\title{
WSGP 5
}

\section{Robert A. Wolak}

\section{On transverse structures of foliations}

In: Zdeněk Frolík and Vladimír Souček and Jiří Vinárek (eds.): Proceedings of the Winter School "Geometry and Physics". Circolo Matematico di Palermo, Palermo, 1985. Rendiconti del Circolo Matematico di Palermo, Serie II, Supplemento No. 9. pp. [227]--243.

Persistent URL: http://dml.cz/dmlcz/701401

\section{Terms of use:}

(C) Circolo Matematico di Palermo, 1985

Institute of Mathematics of the Academy of Sciences of the Czech Republic provides access to digitized documents strictly for personal use. Each copy of any part of this document must contain these Terms of use.

This paper has been digitized, optimized for electronic delivery and stamped
with digital signature within the project DML-CZ: The Czech Digital Mathematics
Library http://project.dml.cz


ON TRATSVERSE STRUCTURES OF FOLIATIONS

Robert A. Wolak

This paper is in final form and no version of it will be submitted for publication elsewhere.

Many authors have considered geometrical structures on the normal bundle of a foliation. It is natural to consider only these structures which are parallel along the leaves of the follation or as some authors say projectible. As examples we can mention bundlelike metrics, transversely projectible or basic connections, transverse symplectic structures, and in general transverse G-atructures. Various properties of these structures have been shown, very often similar to those well known for corresponding structures on manifolds. In th1s paper we shall endearour to show how to obtain such results in a most general way.

Let $M$ be a smooth manifold of dimension $n$, and $F$ a codimension $q$ foliation on $M$ defined by a cocycle $\left\{U_{i}, f_{1}, B_{1 j}\right\}$ where $\left[U_{1}\right]$ form an open covering of $M, f_{1}: U_{1} \rightarrow R^{q}$ is a submersion, and $g_{1 j}: I_{j}\left(U_{i} \cap U_{j}\right) \rightarrow f_{i}\left(U_{i} \cap U_{j}\right)$ is a diffeomorphism such that $I_{j}\left|U_{I} \cap U_{j}=B_{j 1} f_{i}\right| U_{1} \cap U_{j}$. Let $M_{F}$ be a smooth $q$ dimensional manifold equal to $\bigcup_{1} f_{1}\left(U_{1}\right)$. Then the mappings $g_{1 j}$ can be considered as local diffeomorphisms of the manifold $M_{\mathrm{p}}$ and the foliation $F$ as modelled on $M_{F}$. If the manifold 4 is compact we can take a fintte set of indices. Analogously as normal bundles of order $r$ (cf.[7]), we can define transverse $(p, r)-v e l o c 1$ ties and transverse $A$-bundles.

Example 1. Transverse $(p, r)$-velocities ( $p^{r}$-jets). Let $m$ be a point of the manifold $M$. Let $f^{\prime}\left(R^{p}, 0\right) \rightarrow(M, m)$ 
be any looal smooth mapping of $R^{p}$ mapping 0 into $m$. Let $I, 8$ be two such mapplngs and let $(U, \varphi)$ be an adapted ohart such that $\varphi_{:} \mathrm{U} \rightarrow \mathrm{R}^{\mathrm{n}-\mathrm{q}_{2 \mathrm{R}} \mathrm{q}}, \varphi(x)=\left(\varphi_{1}(x), \varphi_{2}(x)\right)$, thus $\varphi_{2}$ 1s oonstant along the leaves. We shall also use the notation

$\varphi_{1}=\left(y_{1}, \ldots, y_{n-q}\right), \varphi_{2}=\left(x_{1}, \ldots, x_{q}\right)$. We say that the mappings 1,8 are equivalent if $f_{0}^{p} \varphi_{2} g=j_{0}^{p} \varphi_{2} f$. This is equivalent to

$$
\partial^{|v|} / \partial x^{v}\left(x_{1} \rho\right)=\partial^{|v|} / \partial x^{v}\left(x_{1} g\right) \text { for any multiindex } v \in \mathbb{d}^{p},|v| \leqslant r
$$

$1=1, \ldots, q$.

We shall denote the number of such indices by $p(r)$ and the set of such indices by $\mathbb{N}(p, r)$. This equivalence relation does not depend on the choice of an adapted ohart at the point in . The equivalence class of a mapping $f$ we denote by $[f]_{p}^{r}$. The set of all equivalence classes at a point m we denote by $\mathbb{M}_{m}^{p, r}(M, F)$, and the space $\bigcup_{m \in \mathbb{M}} \mathbb{M}_{m}^{p, r}(M, F)$ by $\mathbb{N}^{p, r}(M, F)$. By $\pi_{p}^{r}$ let us denote the natural projection of $\mathbb{N}^{p, r}(\mathbb{M}, F)$ into $\mathbb{M}$, i.e. $\pi_{p}^{r}\left([f]_{p}^{r}\right)=$ $=f(0)$. One oan easily check that for any adapted chart $(U, \varphi)$ the set $\bigcup_{m \in U} \mathbb{M}_{m}^{p, r}(\mathbb{M}, F)$ is isomorphic to $U \times R^{q \cdot p(r)}$ and that the Isomorphism is given by the mapping $[\rho]_{p}^{x} \longmapsto\left(\partial^{j v} / \partial x^{v}\left(x_{1} \rho\right)\right) v_{1}^{v}=1, \ldots, q$ Thus, if we denote the mapping defined above by $\varphi_{\mathrm{p}}^{\mathrm{r}}$, $\varphi_{p}^{r} \ddot{i}\left(\pi_{p}^{r}\right)^{-1}(U) \rightarrow R^{n-q} \times R^{q} \times R^{q \cdot p(r)}$, the collection of all such $\varphi_{p}^{I}$ defined by an adapted atlas on $H$, defines an atlas on the space $\mathbb{N}^{\mathrm{p}, \mathrm{T}}(\mathbb{M}, F)$. To see that, one has only to notioe that if $\varphi_{1}, \varphi_{j}$ are two adapted charts for a follated manifold $\left(M_{0}, F\right)$, the composition $\varphi_{i} \varphi_{j}^{-1} ; R^{n-q} \times R^{q} \longrightarrow R^{n-q} \times R^{q} \times R^{q}$ is of the form $\left(f_{1}(y, x), f_{2}(x)\right)$, where $y$ denotes the first $n-q$ coordinates, $x$ the last $q, f_{1}: R^{n-q} \times R^{q} \rightarrow R^{n-q}$, and $I_{2}: R^{n-q} \times R^{q} \longrightarrow R^{q}$, then 
$\varphi_{1 p}^{r}\left(\varphi_{j p}^{r}\right)^{-1}: R^{n-q} \times R^{q} \times R^{q \cdot p(r)} \rightarrow R^{n-q} \times R^{q} \times R^{q \cdot p(r)}$ is equal to $\left(f_{1}, T_{p}^{r}\left(f_{2}\right)\right)$, where $T_{p}^{r}\left(f_{2}\right)$ is the mapping of $T_{p}^{r}\left(R^{q}\right)=R^{q} x R^{q} \cdot p(r)$ Induced by $I_{2}$.

Suming up, we have proved that $\mathbb{1}^{p, r}(M, F)$ is locally trivial eibre bundle, whose total space admits a codimension $q \cdot p(r)+q$ follam tion $F_{p}^{r}$ projecting by $\pi_{p}^{r}$ onto the initial foliation $F$.

If $\mathrm{p}=\mathrm{n}$ and we take only local diffeomorphisms of $R^{n}$ into $M$, the above construction gives a bundle called the transverse frame bundle of the follated manfold $(M, F)$ and denoted by $I^{r}(M, F)$, which is a prinoipal fibre bundle with the fibre $I_{q}^{r}$.

Example 2. The bundle of transverse A-points of (M,F).

Let $A$ be an associative algebra over the field $R$ with the unt 1. The algebra A is called local if it is commutative, of finite dimension over $R$, and if it admits the unique maximal ideal $m$ of codimension 1 euch that $m^{\mathrm{h}+1}=0$ for some non-negative integer h. The smallest such an $h$ is called the height of $A$. Let $R[p]=$ $=R\left[x_{1}, \ldots, x_{p}\right]$ be the algebra of all formal power series in $x_{1}, \ldots, x_{p}$, and let $n r_{p}$ be the maximal ideal of $R[p]$ of all for mal power series without constant terms. Let $a$ be a non-trivial ideal of $R[p]$ such that $R[p] / a$ is of fintte dimension. Then $A=R[p] / a$ is a local algobra with the maximal ideal $m e=n / p / a$. Any local algebra is 1somorphic to such a local algebra (c1.[3]).

Let $C_{m}^{\infty}(M, F)$ be the algebra of the germs of smooth funotions conatant of the leaves of the follations $F$. An algebra homomorphism $\mathcal{L} ; \mathrm{C}_{m}(M, F) \longrightarrow A$ will be called an A-point of $(M, F)$ near to $m$ / or infinitely near transverse point to $m$ of kind $\mathrm{A} /$ if $\alpha(f)=f(m) \bmod m$ for every $f \in C_{m}^{\infty}(M, F)$. We denote by $A_{m}[M, F]$ the set of all A-points of $(M, F)$ near to $m$ and by $A[M, F]=\bigcup_{m \in M} f_{m}[M, F]$. The mapping $\hat{A}_{m}\left[\mathbb{M}_{,} F\right] \nexists_{\mathcal{L}} \longmapsto m \in M$ is denoted by $\pi_{\mathbf{A}^{*}}$

Lot $A=R[p] / a, m e=m r_{p} / a$ and $p_{A}: R[p] \rightarrow A$ be the natural projection. Let us denote by $\mathbb{N}$ the dimension of $m$. Let

$\tau, c_{0}^{\infty}\left(R^{p}\right) \rightarrow R[p]$ be the natural mapping, where $c_{0}^{\infty}\left(R^{p}\right)$ denotes 
the set of germs of smooth functions on $R^{p}$ at 0 .

Definition. Let 1,8 be two smooth mappings of $R^{p}$ into $M$ such that $f(0)=g(0)=m$. We shall say that $f$ is A-equivalent to $g$ at $m i f \tau(h f)=\tau(h g) \bmod m$ for any $h \in C_{m}^{\infty}(M, F)$. By $[f]_{A}$ we denote the equivalence class of $f$, by $f_{m}(M, F)$ all equivalence classes at $m$, and $A(M, F)=\bigcup_{m \in M} A_{m}(M, F)$.

Let $[f]_{A} \in \mathcal{f}_{m}(M, F)$. then $\mathscr{L}_{f}(h)=p_{A}(h f)$ is an A-point of $(M, F)$ near $m$, where $h \in C_{m}^{\infty}(M, F)$. This correspondence $A(M, F) \ni[f]_{A} \longmapsto \alpha_{f} \in A[M, F]$ is a bijection. The proof of this is the same as of Lemma 1.8 of [3].

Let $(U, \varphi)=\left(U,\left(y_{1}, \ldots, y_{n-q}, x_{1}, \ldots, x_{q}\right)\right)$ be an adapted chart, let $b_{1}, \ldots, b_{N}$ be a basis of $m$. On the set $\pi_{A}^{-1}(U)$ we can define the following chart \&

Let $\mathcal{L}$ be an A-point of $(M, F)$ near to $(y, x)$. Then $\mathcal{L}\left(x_{1}\right)=$ $=\sum_{k=1}^{N} a_{i}^{k} b_{k}+x_{i}$. Put

$$
\varphi_{A}(\alpha)=\left(y, x,\left(a_{1}^{k}\right)_{1=1, \ldots, q}^{k=1, \ldots, \pi}\right)
$$

Using the same methods as in Lemma 1.9 and 1.10 of [3] we can check that $\varphi_{\mathrm{A}}$ is bijective.

Let $(U, \varphi)$ and $(V, \psi)$ be two adapted charts and let

$$
\varphi \circ \psi^{-1}: \psi\left(U_{\cap} V\right) \longrightarrow \varphi\left(U_{\cap} V\right) \text { be of the form }\left(f_{1}, f_{2}\right)
$$

where $f_{1}: R^{n} \rightarrow R^{n-q}, f_{2}: R^{q} \rightarrow R^{q}$. Then $\varphi_{A} \circ \psi_{A}^{-1}=$ $=\left(f_{1}, A\left(f_{2}\right)\right) /$ for the definition of $A(f)$ see[3]/. Let $U=\left(U_{1}, \varphi_{1}\right)$ be an adapted atlas of the manfold $(M, F)$. With the differentiable structure defined by $\left(\pi_{A}^{-1}\left(U_{1}\right), \varphi_{1 A}\right)$, the set $A[M, F]$ is a smooth manifold, $\pi_{A}: A[M, F] \rightarrow M$ a fibre bundle over $M$ with the fibreA. On the manifold $A[M, F]$ there is a canonically defined follation $F_{A}$ of the same dimensional as the follation $P$. The profection $\pi_{A}$ maps leaves of the follation $F_{A}$ onto leaves 
of the foliation $F$. The bundle admits a global section, the zero section $8_{0}$. For anj transverse mapping $f: M_{1} \longrightarrow M_{2}$ to the foliation $F$ of the manffold $\mathbb{K}_{2}, I$ defines a smooth mapping $A(f): A\left[M_{1}, f^{H} F\right] \rightarrow A\left[M_{2}, F\right]$,

$A(f)(\alpha)(h)=\alpha(f h)$, where $\alpha \in A_{m}\left[M_{1}, f^{x} F\right]$ and $h \in C_{m}^{\infty}\left(M_{2}, F\right)$.

If $F$ is the follation by points of the manifold $M, A[M, F]=$ - A[M]- the bundle of A-points of M.

Example 3. Transverse natural bundles.

Let Pol $_{q}$ be the category of smooth manifolds follated by smooth codimension $q$ follations with smooth, follation preserving transverse mapping to the foliation.

Definition. A covariant functor $N$ on the oategory Fol $q$ into the category of locally trivial fibre bundles and their fibre mappings Is called a transverse natural bundle if the following conditions are satisfied: $i / f o r$ anj foliated manifold $(M, F), \mathbb{N}(M, F)$ is a fibre bundle over the manifold $M$;

$$
\text { ii/if } f_{8}\left(M_{0}, F_{0}\right) \longrightarrow\left(M_{1}, F_{1}\right) \text { is a transverse mapping }
$$

such that $f^{n} F_{1}=F_{0}$, then $N(f): N\left(M_{0}, F_{0}\right) \rightarrow N\left(M_{1}, F_{1}\right)$ covers $f$ and maps the fibre $\mathbb{N}\left(M_{0}, F_{0}\right) x$ over $x$ diffeomorphically onto the fibre $N\left(M_{1}, F_{1}\right) f(x)$ over $f(x)$;

iii) Is is a regular funotor 1.e. If $f$ \& UXM $\rightarrow M_{1}$ is a differentiable mapping, $U$ an open subset of $\mathrm{R}^{\mathrm{k}}$, such that for any point $t$ of the set $U$, the mapping $e_{t^{2}}\left(M_{0}, F_{0}\right) \rightarrow\left(M_{1}, F_{1}\right)$ $f_{t}(x)=f(t, x)$ is a transverse mapping to the foliation and $P_{t}^{N} F_{1}=F_{0}$, then the mapping $\operatorname{UxN}\left(M_{0}, F_{0}\right) \exists(t, y) \mapsto \mathbb{N}\left(P_{t}\right)(y) \in \mathbb{N}\left(M_{1}, F_{1}\right)$ 18 of class $\mathrm{c}^{\infty}$.

Properties, 1/ For any morphism $f:\left(M_{0}, F_{0}\right) \rightarrow\left(M_{1}, F_{1}\right)$ the fibre bundle $P^{N}\left(M_{1}, F_{1}\right)$ is lsomorphic to $\mathbb{N}\left(M_{0}, F_{0}\right)$.

$2 /$ Let 1,8 be two morphisms of $\left(M_{0}, F_{0}\right)$ into $\left(M_{1}, F_{1}\right)$ such that $f(m)=g(m)$. Iet $(0, \varphi)$ be an adapted chart at $m$ and $(V, \psi)$ an adapted chart at $f(m)$. Since the mappings $f$ and $g$ preserve the foliation, the mappings $\bar{f}=\psi \rho \varphi^{-1}, R^{n-q} \times R^{q} \rightarrow R^{m-q} \times R^{q}$, 
$\dot{\bar{B}}=\psi_{B} \varphi^{-1}, R^{n-q} \times R^{q} \longrightarrow R^{m-q_{x R}}$, where dim $M_{0}=n, \operatorname{dim} M_{1}=m$, are of the form $f(y, x)=\left(f_{1}(y, x), f_{2}(x)\right), g(y, x)=$ $=\left(g_{1}(y, x), g_{2}(x)\right)$, where $y$ denotes the first $n-q$ coordinates $x$ the last $q$ coordinates, $f_{1}, g_{1}, R^{n} \rightarrow R^{m-q}$ and $f_{2}, B_{2}: R^{n} \rightarrow R^{q}$. If the germs of the mappings $f_{2}$ and $g_{2}$ at

$\varphi^{-1}(\mathrm{~m})$ are equal, then the mappings $\mathbb{N}(f)$ and $\mathbb{N}(g)$ define the same mapping on the fibre $\mathbb{N}\left(M_{0}, F_{0}\right)_{m}$ •

Proof. The property 1 is obvious. One has only to show the second. Let $(U, \varphi),(V, \psi)$ be two adapted charts such that $f_{2}\left|\varphi(U)=g_{2}\right| \varphi(U)$ and $\varphi(U)=D^{n-q} \times D^{q}$, where $D^{k}$ denotes the k-disc. Assume that $\varphi(m)=0$ and $\psi(f(m))=0$. Then the following diagram is commutative.

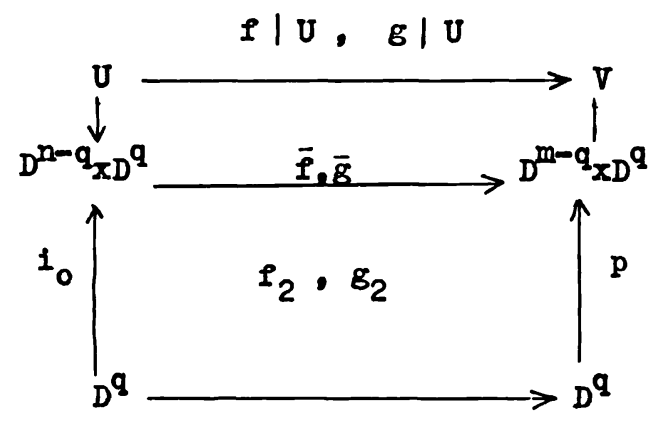

where $I_{0}: D^{q} \longrightarrow D^{n-q} \times D^{q}$ is given by $1_{0}(x)=(0, x)$ and $p: D^{n-q} \times D^{q} \longrightarrow D^{q}$ by $p(y, x)=y$.

Since IN is a functor, it is oufficient to show that the mappings 1 , and $B$ Induce the same mapping in the fibre over 0 . But since $p \bar{\rho} I_{0}=I_{2}$ and $\bar{p} \bar{g} I_{0}=g_{2}, N\left(f_{2}\right)=\mathbb{N}(p) \mathbb{N}(\bar{f}) \mathbb{N}\left(I_{0}\right)=\mathbb{N}\left(g_{2}\right)=$

$=\mathbb{N}(p) \mathbb{N}(\overline{\mathrm{B}}) \mathbb{N}\left(1_{0}\right)$ - But $\mathbb{N}(p)$ and $\mathbb{N}\left(1_{0}\right)$ induce isomorphisms on the fibre, hence the mappings $\mathbb{N}(\bar{g})$ and $\mathbb{N}(\bar{f})$ are equal on the flbre over 0 , which ends the proof.

Definition. A transverse normal bundie If is finite order $r$ if for ans two morphisms $P, B,\left(M_{0}, F_{0}\right) \longrightarrow\left(M_{1}, F_{1}\right)$ the integer $r$ is the smallest one for whioh the following implication is trues 


$$
f_{x}^{r}=f_{\frac{x}{x}}^{r} \Rightarrow \mathbb{N}(f)(y)=\mathbb{N}(g)(y) \text { for any point } y \text { of }
$$

the eibre $N\left(M_{0}, F_{0}\right)_{x}$.

Having this defintion we can prove the following theorem.

Theorem 1. Iet $\mathbb{N}$ be a transverse natural bundle. Then there exists an integer $r$ and an $I_{q}^{r}$-space $W$ such that II is 1somor phic to the fibre bundle associated to the transverse rmeme bundle with the standard fibre W. The smallest such integer $r$ is the order of the transverse natural bundle $\mathbb{N}$.

Proof. The first case to consider is that of a follated manifold (M,F) whose foliation $F$ is given by a global submersion $I: M \rightarrow M_{0}$. In this case the transverse rmerame bundle $I^{r}(M, F)$ is isomorphic to $f^{n} I^{r}\left(M_{0}\right)$. Let $B\left(I^{r}\left(M_{0}\right), W\right)$ be an associated Iibre bundie to the reframe bundle $I^{I}\left(M_{0}\right)$ with the standard IIbre $W$. Then $P^{x} B\left(I^{r}\left(M_{0}\right), W\right)$ is an as800iated fibre bundle to the transverse r-ersme bundle $I^{Y}(M, F)$ with the standard Ilbre $W$. In what follows we shall denote $B\left(I^{r}\left(M_{0}\right), W\right)$ by $B\left(H_{0}, W\right)$ and $f^{\prime} B\left(I^{r}\left(M_{0}\right), W\right)$ by $B(M, F ; W)$. The 1somorphism from Palais-Terg's Theorem (cf.[6]) we shall denote by $B\left(M_{0}\right)$. Thus the following diagram is commutative:

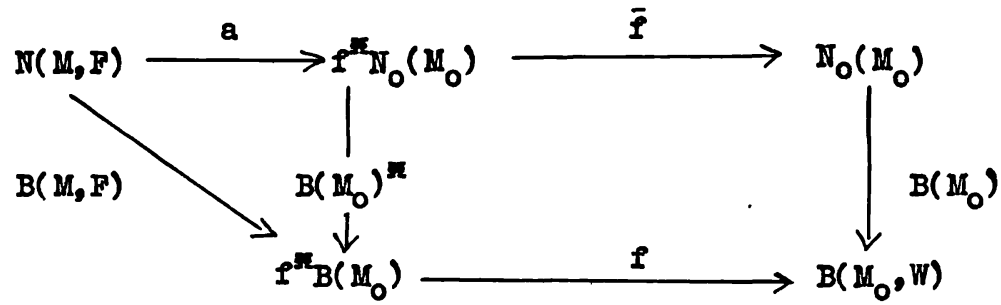

where $B\left(X_{,} F\right)=B\left(M_{0}\right)^{m} a_{\text {, a }}$ is the isomorphism Iram Property 1 and $N_{0}$ is the natural bundle on the category of $q$ manifolds obtained from $I$ by follating $q$ manifolds by points. To this natural bundle we have applied Palais-Terng's Theorem. The mapping $B(U, F)$ is an 1somorphism of fibre bundles since both mappings $B\left(M_{0}\right)^{n}$ and a are. .

To complete this special case we have to show that these lsomor phlams are functorial. Let. $F_{1}$ be a foliation given by a global submersion $f_{1}: \mathrm{H}_{1} \longrightarrow \nabla_{1}$ and $F_{2}$ be a follation given by a 
global submersion $\mathrm{I}_{2}: \mathrm{M}_{2} \longrightarrow \mathrm{V}_{2}$ such that the diagram

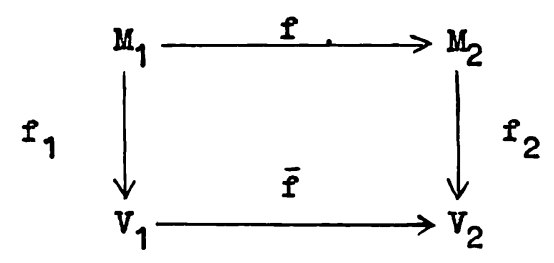

is commutative for some smooth mapping $f$. We have to show that the following diagram is commutative.

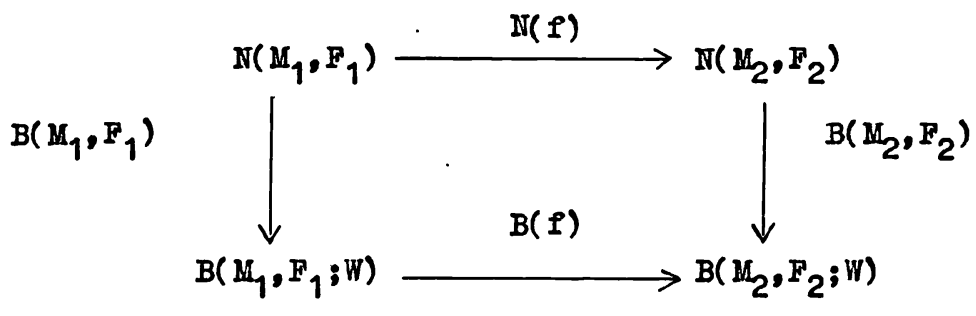

It is so, because the following diagram is commutatives

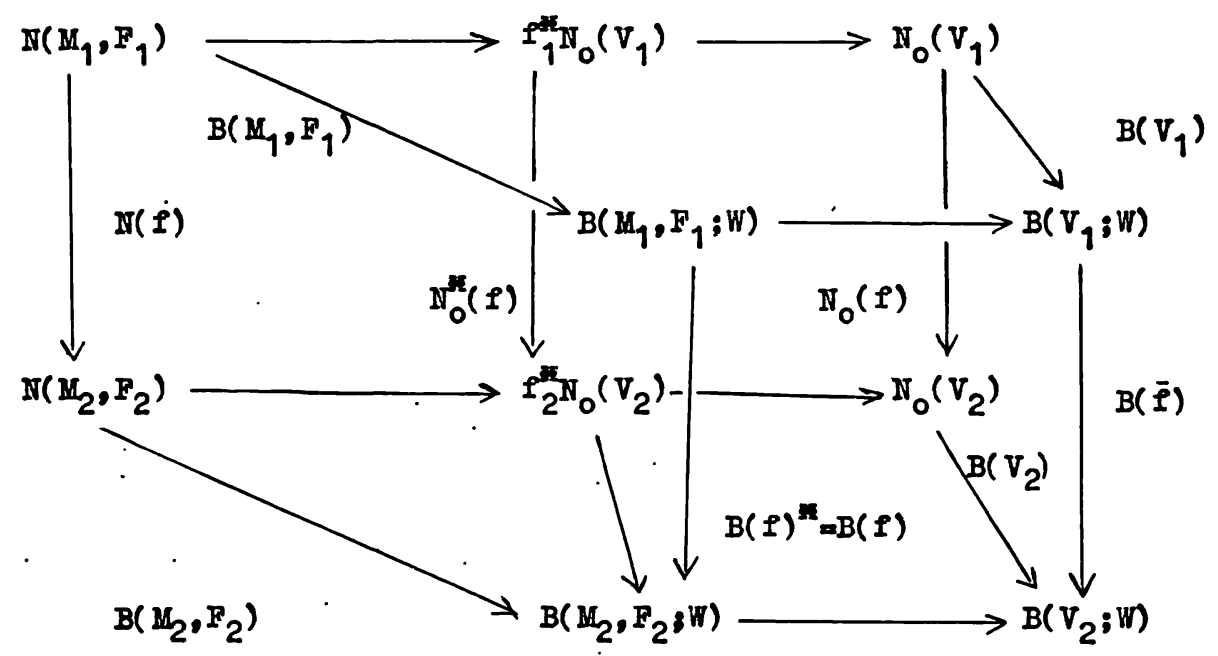

The general case can be proved in the following way. Iet $(M, F)$ be a follated manifold. Then there exists a covering $U=\left\{U_{\alpha}\right\}_{A}$ and global submersion $f_{\alpha}: U_{\alpha} \rightarrow V$ defining the follation 
$F \mid U_{\alpha}$. Since $\mathbb{N}\left(U_{\alpha}, F \mid U_{\alpha}\right) \simeq \mathbb{N}(\mathbb{M}, F) \mid U_{\alpha}$, the bundle $\mathbb{N}(\mathbb{M}, F)$ is isomorphic to $\frac{\|}{\alpha \in A} \mathbb{N}\left(U_{\alpha}, F \mid U_{\alpha}\right) / \sim$, where the relation $\sim$ is induced by the natural identification of points in various $U_{\mathscr{L}}$. For each 2 we have the isomorphism $B\left(U_{\alpha}, F \mid U_{\alpha}\right), \quad B\left(U_{\alpha}, F \mid U_{\alpha}\right)$ \& : $\mathbb{I}\left(U_{\alpha}, F \mid U_{\alpha}\right) \longrightarrow B\left(U_{\alpha}, F \mid U_{\alpha} ; W\right)$, which agrees with the relation $\sim$ and the corresponding relation for the bundles $B\left(U_{\alpha}, F \mid U_{\alpha} ; W\right)$, $\alpha \in A$. Thus the bundle $\frac{1}{\alpha \in A} \mathbb{N}\left(U_{\alpha}, F \mid U_{\alpha}\right) / \sim$ is isomorphic to $\frac{1}{\alpha \in A} B\left(U_{\alpha}, F \mid U_{\alpha} ; W\right) / \sim \quad$ This 1somorphism induced by $B\left(U_{\alpha}, F \mid \Psi_{\alpha}\right)$ we shall denote by $B_{Q}(M, F)$. Since the bundle $\frac{1}{\alpha \in A} B\left(U_{\alpha}, F \mid U_{\alpha} ; W\right) /$ Is isomorphic to the associated fibre bundle to the transverse $r$-Prame bundle of (M,F) with the standard fibre $W, B(M, F ; W)$, for each such covering $\mathcal{U}=\left\{U_{\alpha}\right\}$ we have defined an isomorphism of $\mathbb{I}(M, F)$ onto $B(M, F ; W)$ denoted by $B U(M, F)$. We have to show that this isomorphism does not depend on the covering $U_{\text {. Let }} U_{6}$ and $\psi$ be two such coverings and $W^{\psi}$ be a covering which is finer then $\mathcal{U}$ and $\mathcal{W}$. Then the following diagram is comutative.

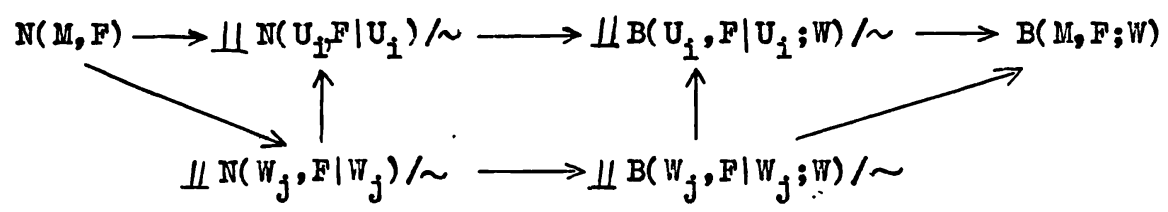

where the horizontal arrows have been defined previously, and the two vertical arrows are naturally defined. Thus $B_{q u}(M, F)=B_{q u}(M, F)$. Therefore these 1somorphisms are independent of the choice of a covering $Q$, and we denote the isomorphism thus obtained by $B(M, F)$

The only remaining thing to prove is to show that $B(\mathbb{M}, F)$ define an isomorphism of the functors $N$ and $B(\cdot ; W)$.

Let $\mathrm{P}_{8}\left(\mathrm{H}_{1}, \mathrm{~F}_{1}\right) \longrightarrow\left(\mathrm{M}_{2}, \mathrm{~F}_{2}\right)$ be a morphism. We can choose a covering $U=\left\{U_{1}\right\} I$ of $\mathbb{u}_{1}$ with submersions $g_{1}: U_{1} \rightarrow v_{1}$ defining the follation $F_{1}$, and a covering $W^{N}=\left\{W_{j}\right\} J$ of $M_{2}$, with submersions $h_{j}: W_{j} \rightarrow z_{j}$ defining the foliation $F_{2}$ such that for any $i \in I$ there exists $j \in J$ with the property: $f\left(U_{1}\right) \subset W_{j}$. Let us denote $f \mid U_{i}: U_{1} \rightarrow W_{j}$ by $f_{I}$. Then $f_{I}$ induces a mapping $\bar{f}_{1}: V_{1} \rightarrow Z_{j^{*}}$ Because $\mathbb{N}$ and $B(\cdot, W)$ are functors, the diagrams 


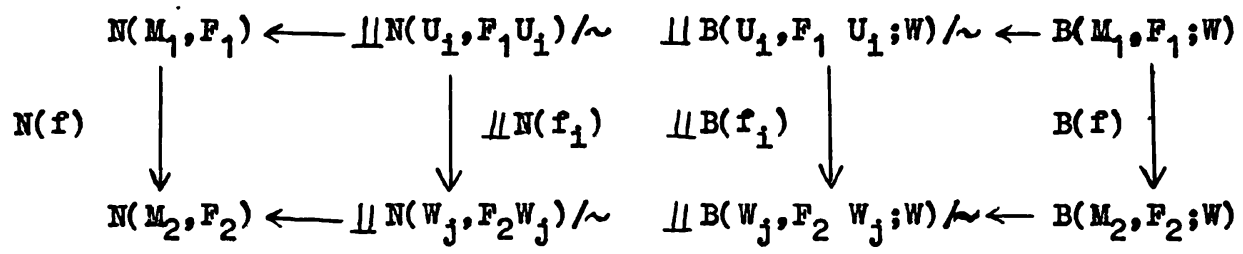

are commutative.

Because the follations $F_{1} \mid U_{1}$ and $F_{2} / W_{j}$ are globally defined by submersions, it follows from the first part of the proof that the diagram

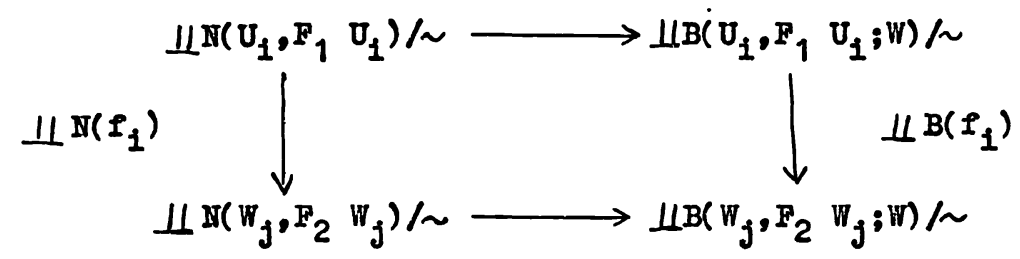

commutes, which effectively ends the proof.

From our point of view, the bundles considered in Examples 1,2,3 are nothing else but the inverse images of the $(p, r)$-velooities, A-bundles and natural bundles, respectively, on the manifold $M_{p}$ via the mappings $f_{1}$. They glue together because diffeomorphlsms can be lifted to these bundles. Thus for these structures and geometrical objects connected with them we have the following ndictionary". On the left hand alde there are transverse objects on the foliated manifold $(M, F)$, and on the right hand side there are corresponding objects on the manifold $M_{\mathrm{P}}$.

normal bundle of order $r$

bundle of transverse $(p, r)$-relocities bundle of transverse A-points transverse natural bundle

follated $(p, r)$-tensor

transversily projectible a-structure basic $r-f o r m$

basic conneotion tangent bundle of order $r$ bundle of $(p, r)$-velocities bundle of A-points natural bundle $(p, r)$-tensor G-structure reform connection

One can lift follated tensors and basic connections to the transverse bundles mentioned above by repeating the constructions for 
the corresponding bundles on manifolds with only minor changes. But this process is very tedious (cf. [7]). Using the correspondence explained in the "dictionary" we can prove it in the following waj. Let $t$ be a transverse object on $(M, F)$. If we can lift such an object to a transverse bundle $B$ of the type considered, the corresponding object on the manifold $M_{F}$ can be lifted to the cor responding bundle on the manifold $M_{F}$, and this lift is left invariant by the lifts of the transformations $B_{1 j}$, since any transverse object projected onto $M_{F}$ ls left invariant by $B_{1 j}$. Inver sely, any object on $M_{F}$ invariant by $g_{i j}$ can be lifted to $(M, F)$ Therefore we have to check the following. Let $t$ and $t$ ' be two objects of a given type on manffolds $N$ and $N^{\prime}$, respectively, and $f$ be a diffeomorphism of $N$ onto $\mathbb{N}^{\prime}$, such that $t=f^{\prime} t^{\prime}$. Let $I$ be the lifting considered. Then $I(t)=I(f)^{n} I\left(t^{\prime}\right)$.

First, we shall use this procedure to lift follated tensor fields. Let $t$ be a follated tensor field of type $(p, s)$ on the follated manifold $(M, F)$. Then $t$ defines a tensor field $\tilde{t}$ of type $(p, s)$ on the manifold $M_{F}$ such that $t \mid U_{i}=f_{i}^{\tilde{t}}$ and $g_{i j}^{n} \tilde{t} \mid f_{j}\left(U_{i} \cap U_{j}\right)=$ $=\tilde{t} \mid \rho_{1}\left(U_{1} \cap U_{j}\right)$.

We shall lift follated tensor fields to the following transverse bundles.

1/ Normal bundle of order $r$.

The normal bundle $\mathbb{N}^{r}(M, F)$ of order $r$ admits a foliation $F^{r}$ modelled on the manifold $T^{r}\left(M_{P}\right)$ with transformations $T^{r}\left(g_{1 j}\right)$. To these bundles we shall be able to lift any follated tensor fleld; thus we have to check

$$
\left(f^{n}\right)^{(\lambda)}=\pi^{x}(1)^{x_{t}}(\lambda) \text { for } \lambda=0,1, \ldots, x \text {. }
$$

11/ Bundle of transverse $(p, r)$-relocities.

The bundle of transverse $(p, r)-\nabla e l o c i t i e s ~ N^{p, r}(M, F)$ on the foliated manifold $(M, F)$ has a natural foliation $F_{p}^{r}$. The foliation $F_{p}^{r}$ is modelled on $T^{p, r}\left(M_{F}\right)$ with $T_{p}^{r}\left(g_{1 j}\right)$ as transformations. Therefore to be able to lift follated tensor fielde we have to check that if $f^{x} t=t^{\prime}$ then $T_{p}^{r}(f)^{x} t^{(\lambda)}=t^{(\lambda)}$ for ans $\lambda \in N(p, r)$.

Ii1/ Bundle of transverse A-points .

The bundle $A(M, F)$ of transverse A-points of the follated manifold (N,F) admits a foliation $F_{A}$ modelled on the manifold $A\left(M_{P}\right)$ with transformations $A\left(B_{1 j}\right)$. To these bundles we shall lift follated 
tensor fields of type $(0,8)$ or $(1,8)$; thus we have to check

$$
\left(f^{\pi} t\right)^{(\lambda)}=A(f)^{\pi_{t}^{(\lambda)}} \text { for } \lambda=0, \ldots, N
$$

iv/ Pransverse natural bundles.

Let $N\left(M_{0} F\right)$ be a transverse natural bundle on the follated manifold $(M, F)$ - Then the manifold $\mathbb{N}(M, F)$ admits a foliation $F_{\mathbb{N}}$ of the same dimension as $F$ modelled on $\mathbb{N}\left(M_{F}\right)$ with transformations - $N\left(g_{i j}\right)$. We shall be able to lift follated tensors of type $(1, \theta)$

" but only if the functor $N$ fulfils the following condition:

(0) The union of all open orbits of the action of $I_{q}^{r}$ on the standard fibre $W$ is dense in $W$.

The condition $(0)$ allows to define the complete lift of tensor fields of type $(1,8)$ to this natural bundle (cf. [1]).

Because the lifts for the first three types are defined multiplicatively, we have to check the equalities only for functions, 1-forms and vector fields. The case of natural bundles will be dealt separately.

a/ Let $h \in C^{\infty}(M), f: M \rightarrow M$ be a local diffeomorphism. We have to show that
$1 /$
$(h \rho)^{(\lambda)}=h^{(\lambda)} T^{r}(f)$
for $\lambda=0, \ldots, r$;
$11 /$
$(h f)^{(\lambda)}=h^{(\lambda)} T_{p}^{r}(f)$
for $\lambda \in \mathbb{N}(p, r)$;
i11/
$(h f)^{(\lambda)}=h^{(\lambda)} A(f)$
for $\lambda=0, \ldots, \mathbb{N}$.

The equalities follow directly from the definitions.

b/ Let $X$ be a vector field on $M, f: M \rightarrow M$ be a local diffeomorphism .

We have to show that
1/

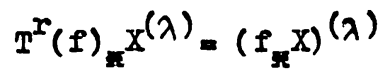
for $\lambda=0, \ldots, r$;
ii/

$$
T_{p}^{x}(f)_{n} X^{(\lambda)}=\left(f_{n} X\right)^{(\lambda)}
$$
for $\lambda \in \mathbb{N}(p, r)$;
1ii/

$$
A(f)_{x^{x}} x^{(\lambda)}=\left(f_{x} X\right)^{(\lambda)} \quad \text { for } \quad \lambda=0, \ldots, \mathbb{N} \text {. }
$$

Since the proofs of these equalities are similar, we shall show 
it only for A-bundles.

$$
\begin{aligned}
& A(\rho)^{x_{w}(\lambda)}\left(X^{(\gamma)}\right)=w^{(\lambda)}\left(A(\rho) x_{x} X^{(\gamma)}\right) \\
& =w^{(\lambda)}\left(\left(e_{z} X\right)(\gamma)\right. \text {, } \\
& =\sum c_{\lambda r}^{\mu}\left(w\left(f_{X} X\right)\right)^{(\mu)} \\
& =\sum c_{\lambda r}^{\mu}\left(f^{\mu x} w(X)\right)^{(~)} \\
& =\left(f^{x} w\right)^{(\lambda)}\left(x^{(\gamma)}\right)
\end{aligned}
$$

for any vector field $x, \lambda, v=0, \ldots, N$. Therefore $A(f)^{n} w^{(\lambda)}=$ $=\left(f^{n} w\right)(\lambda)$.

Let $t$ be a foliated tensor field on the manifold $(\mu, F)$, and let $t^{(\mu)}$ be the lift to one of the considered bundles obtained in the above way. Then the tensor field $t^{(\mu)}$ has the following properties.

$$
\begin{aligned}
& \text { if } \quad \mathcal{L}_{X^{(\lambda t}}^{k}(\mu)=\left(\mathcal{L}_{X^{t}}^{t}\right)^{(\mu+\lambda-r)} \quad \text { for } \lambda, \mu=0, \ldots, r \text {; } \\
& \text { i1/ } \quad \mathcal{L}_{X K \lambda\rangle t}^{k}(\mu)=\left(\mathcal{L}_{X}^{k} t\right)^{(\mu-\lambda)} \quad \text { for } \mu, \lambda \in \mathbb{N}(p, r) \text {; } \\
& \text { ii1/ } \mathcal{L}_{\mathrm{X}^{(\lambda) t}}^{\mathrm{k}}{ }^{(\mu)}=\sum_{c_{\lambda \mu}^{V}}\left(\mathcal{L}_{\mathrm{X}}^{\mathrm{k}} t\right)^{(v)} \text { for } \lambda, \mu, \gamma=0, \ldots, \mathbb{N} \text {, }
\end{aligned}
$$

where $X$ is a follated vector fleld and $\mathcal{L}^{k}$ denotes the contraction. The equalities follow from the corresponding equalities for tensor fields on model manifolds. It is also clear that if a lifting of a tensor field fulfilg suitable equality, it must be unique as the vector fields $x^{(\lambda)}$ span the whole tangent space. In this way we retrieved the results of lifting of follated tensor fields to normal bundles of order $r$ contained in [7] and proved the following.

Theorem 2. Iet $(M, F)$ be a follated manifold.

Then for any $\lambda \in \mathbb{N}(p, r)$, there exists the unique lifting

I $\lambda: T_{v}^{E}(M, F) \longrightarrow T_{v}^{E}\left(\mathbb{N}_{p}^{r}(M, F), F_{p}^{r}\right), E=0,1$ such that

$$
\mathcal{L}_{\mathrm{X}}^{\mathrm{k}\langle\omega} \mathrm{L}_{\lambda} t=I_{\lambda-\mu}\left(\mathcal{L}_{X}^{\left.k_{t}\right)} \text { for any } \mu \in \mathbb{N}(p, r)\right. \text {, }
$$


and for ans $\lambda=0, \ldots, \mathbb{N}$, there exists the unique lifting

$$
\begin{aligned}
& \left.I_{\lambda}: T_{V}^{E}(M, F) \longrightarrow T_{V}\left(A(M, F), F_{A}\right)\right), E=0,1 \text {, such that } \\
& \mathcal{L}_{X^{(\mu)}}^{k}{ }^{I}{ }^{t}=\sum{ }^{c}{ }_{\lambda}^{v} \mu_{V}^{I_{V} t} \quad \text { for any } \mu=0, \ldots, \pi \text {. }
\end{aligned}
$$

Now, we shall deal with the fourth case. For transverse natural bundles the following theorem is true.

Theorem 3. Let ( $M, F)$ be a follated manifold, II a transverse natural bundle fulfilling the condition ( 0 ). For ans follated tensor field $t$ of type $(1,8)$, there exists the unique lift $t^{c}$ to the total space of the bundle $\mathbb{N}(M, F)$ such that

$$
t^{c}\left(x_{1}^{C}, \ldots, x_{B}^{C}\right)=\left(t\left(x_{1}, \ldots, x_{8}\right)\right)^{c}
$$

for any foliated vector fields $Z_{1}$.

Proof. Let $X$ be a follated vector field and $X^{\prime}$ be its representative. Iet $\varphi_{t}$ be the flow of $x^{\prime}$. It preserves the follation $P$. Thus we can define $\mathbb{N}\left(\varphi_{t}\right)$, which in Its turn is the flow of a vector fleld $\widetilde{X}^{\prime}$ on the total space of $\mathbb{H}(M, F)$, and preserves the foliation $F_{N}$. Thus we have defined a follated reotor fleld on $\mathbb{N}(\mathbb{M}, \mathrm{F})$ which does not depend on the choice of $Z^{\prime}$ and is denoted by $x^{C}$. On an open set $U$ on which the foliation $F$ is defined by a submersion $f, X^{C}$ is an inverse image by $N(f)$ of the complete lift $x_{0}^{C}$ of the oorresponding vector field $x_{0}$. Thus $x^{C}$ can be obtained as the inverse image of the complete lift of a veotor fleld on the model manifold $u_{F}$, as directly from the definition $(f, X)^{C}=\pi(\rho) x_{x} X^{C}$, for any vector fleld $X$ and ans local diffeomorphism $\rho$.

Let $t$ be a tensor fleld of type $(1,8)$ on the manifold $I$ and $f$ be a local diffeomorphism of $N$. Then

$$
\begin{aligned}
& \mathbb{H}(f)^{n_{t}}{ }^{C}\left(x_{1}^{C}, \ldots, X_{B}^{C}\right)=\mathbb{N}\left(f^{-1}\right)_{n}, t^{C}\left(\mathbb{N}(f)_{n} x_{1}^{C}, \ldots, \mathbb{N}(f)_{n} x_{B}^{C}\right) \\
& =\pi\left(f^{-1}\right)^{x} t^{C}\left(\left(f^{x} x_{1}\right)^{C}, \ldots,\left(f^{x} X_{8}\right)^{C}\right) \\
& =N\left(f^{-1}\right)_{x}\left(t\left(f_{x}\left(x_{1}\right), \ldots, f_{x}\left(x_{s}\right)\right)\right)^{C}
\end{aligned}
$$




$$
\begin{aligned}
& =\left(f^{-1} t\left(e_{n} X_{1}, \ldots, f_{x} X_{s}\right)\right)^{C} \\
& =\left(f^{x} t\left(x_{1}, \ldots, x_{s}\right)\right)^{c} \\
& =\left(f^{n} t\right)^{C}\left(x_{1}^{C}, \ldots, x_{B}^{C}\right) \text {. }
\end{aligned}
$$

Thus because of the condition $(0), \mathbb{N}(f)^{x_{t}}=\left(f^{x} t\right)^{C}$. Therefore, each foliated tensor field $t$ of type $(1,5)$ can be lifted to the total space of the transverse natural bundle $N(M, F)$. We put $t^{C} \mid U_{1}=f_{1}^{n} t_{0}^{C}$, where $t_{0}$ is the corresponding tensor field on $f_{1}\left(U_{1}\right)$. Then

$$
\begin{aligned}
t^{C}\left(x_{1}^{C}, \ldots, x_{s}^{C}\right)_{v} & =e_{1}^{k} t_{0}^{C}\left(x_{1}^{C}, \ldots, x_{p}^{C}\right) \\
& =\left(d N\left(f_{1}\right)\right)^{-1} t_{0}^{C}\left(d f_{1}\left(x_{1}\right)^{C}, \ldots, d f_{1}\left(x_{s}\right)^{C}\right) \\
& =\left(d N\left(f_{1}\right)\right)^{-1} t_{0}^{C}\left(x_{10}^{C}, \ldots, x_{80}^{C}\right) \\
& =\left(d\left(N\left(f_{1}\right)\right)\right)^{-1}\left(t_{0}\left(x_{10}, \ldots, x_{s 0}\right)\right)^{C} \\
& =\left(\left(d f_{1}\right)^{-1} t_{0}\left(x_{10}, \ldots, x_{s 0}\right)\right)^{C} \\
& =\left(t\left(x_{1}, \ldots, x_{s}\right)\right)^{C} .
\end{aligned}
$$

where $X_{0}$ is the corresponding vector fleld on $M_{F}$ to the follated vector field $X$. This ends the proof of Theorem 3 .

To complete this short paper we shall prove that the lift of basic connections to normal bundles of order $r$, bundles of transverse $(p, r)$-velocities and transverse $A$-bundles exist. Because we shall apply the same method as for tensor fields, we have to cheok only the following. If $\nabla$ is a connection on a manifold $M, \nabla^{\prime}$ a connection on a manifold $M^{\prime}$, and $f$ is a local diffeomorphism

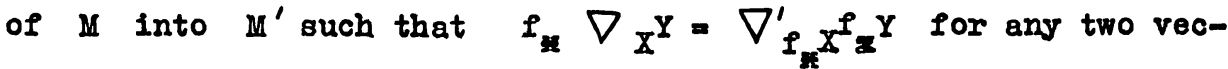
tor fields, then

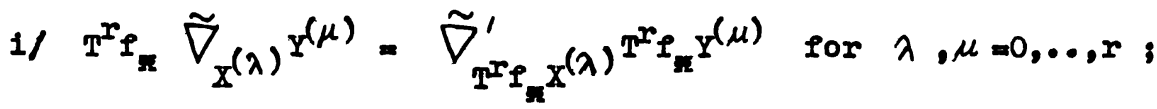

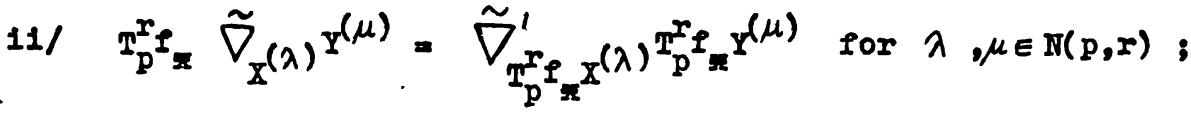




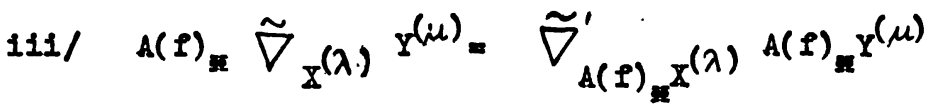

$$
\begin{aligned}
& \text { for } \lambda, \mu=0, \ldots, \mathbb{N} \text {. }
\end{aligned}
$$

where $X, Y$ are vector flelds, and $\tilde{\nabla}$ is the lift of the conneot1on $\nabla$. The proof of these cases are similar. We shall check only the third one.

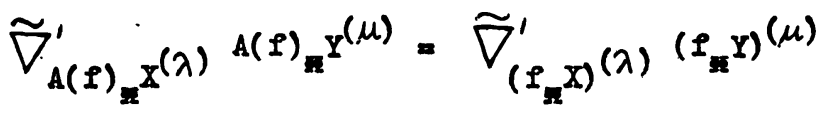

$$
\begin{aligned}
& =\sum_{0}^{0} \gamma_{\mu \mu}\left(\nabla_{P_{n} X}^{\prime} e_{n} Y\right)^{(\gamma)} \\
& =\sum_{\lambda_{\mu \mu}}^{r}\left(f_{n}\left(\nabla_{X}{ }^{Y}\right)\right)^{(r)} \\
& =\sum c_{\lambda \mu}^{V} A(\rho){ }_{s s}\left(\nabla X_{X}^{Y)}(V)\right. \\
& =A(f)_{H}\left(\sum c_{\lambda \mu}^{V}\left(\nabla X^{Y}\right)^{(v)}\right) \\
& =A(\rho){ }_{E}\left(\tilde{\nabla}_{X}{ }^{(\lambda) Y^{(\mu)}}\right) \text {. }
\end{aligned}
$$

Theorem 4. Let ( $M, F)$ be a foliated manifold. Let $\nabla$ be a basic connection. Iet $B(M, F)$ be a transverse A-bundle. Then there exists a connection $\widetilde{\nabla}$, basic for the follation $F_{A}$ of the total space of the bundle $B(M, F)$ such that

$$
\tilde{\nabla}_{X}(\mu){ }^{(\lambda)}=\sum c{ }_{\mu \lambda}^{v}\left(\nabla x^{y}\right)^{(v)} .
$$

for any foliated veotor fields $X, Y$ on the manifold $(M, F)$ and $\lambda, \mu=0, \ldots, \pi$.

\section{REFEREITCES}

1. GANCARZEWICZ J., Complete Iffts of tensor fields of type $(1, k)$ to natural bundles. Prace Mat. 23(1982), 51-84 .

2. MORIMOTO A., Prolongations of Gometric Structures, Lecture Notes, Math. Inst. Nagoja Univ. 1969. 
3. MORTMOTO A., Ifftings of some types of tensor fields and connections to tangent bundles of $\mathrm{p}^{\mathrm{r}}$-relocities, Nagoya Math. $\mathrm{J}$. 40(1970), 13-31.

4. MORIMOTO A.,Ifftings of tensor fields and connections to tangent bundles of hlgher order, Nagoya Math. J.40(1970), 85-97.

5. MORIMOTO A., Prolongation of connections to bundles of infinitesimal near points, J.Diff.Geom.11(1976), 489-498.

6. PALAIS R.S.,TERWG C.-I., Natural bundles have finite order, TOpology 16(1977), 271-277.

7. WOIAK R., Normal bundles to follations of order $r$, preprint.

INSTYTUT MATEMATYKI

UNIWERS YTET JAGIELLORSKI

UL. REYMONTA 4

30-059 KRAKסW 